PSICOLOGÍA

IBEROAMERICANA
Psicología Iberoamericana ISSN: 1405-0943

revista.psicologia@ibero.mx

Universidad Iberoamericana, Ciudad de México México

\title{
Cómo vivencian los psicoterapeutas en formación el inicio de su entrenamiento y la supervisión
}

Vethencourt D'Escrivan, Veronica

Cómo vivencian los psicoterapeutas en formación el inicio de su entrenamiento y la supervisión

Psicología Iberoamericana, vol. 26, núm. 1, 2018

Universidad Iberoamericana, Ciudad de México, México

Disponible en: http://www.redalyc.org/articulo.oa?id=133959553008 


\section{Cómo vivencian los psicoterapeutas en formación el inicio de su entrenamiento y la supervisión}

How psychotherapists in training experience the beginning of training and supervision

Veronica Vethencourt D'Escrivan veronica.vethencourt@gmail.com Universidad De Las Américas, México

Resumen: La vivencia emocional asociada al inicio del entrenamiento práctico de psicoterapeutas no recibe la atención que requiere, considerando que la misma puede incidir negativamente en los resultados de la psicoterapia y en el estado mental de los psicoterapeutas. Esta investigación cualitativa busca explorar la vivencia emocional de 13 terapeutas en entrenamiento y de dos supervisores/docentes relacionada al inicio del entrenamiento. Los resultados obtenidos muestran la dificultad inherente al enfrentarse por primera vez con los requerimientos de la formación, y los riesgos que implican para los terapeutas y para sus clientes. El abordaje fenomenológico permite observar la manera en que se vive esta etapa en el desarrollo profesional de psicoterapeutas, lo que es útil en la medida que permite generar un aumento de autoconsciencia sobre los sentimientos y riesgos asociados a este proceso y posibilita así la aplicación de medidas preventivas y correctivas.

Palabras clave: inicio del entrenamiento, supervisión, psicoterapeutas, experiencia emocional, formación.

Abstract: The emotional experience associated with the beginning of the practical training of psychotherapists does not receive the attention it requires, considering that it may have a negative effect on the results of psychotherapy and the mental state of psychotherapists. This qualitative research seeks to explore the emotional experience of 13 therapists in training and two supervisors/teachers related to the beginning of training. The results show the inherent difficulty in facing for the first time the training requirements and risks involved for therapists and their clients. The phenomenological approach allows us to observe the way in which this stage is experienced in the professional development of psychotherapists. This is useful because it increases the selfawareness surrounding the feelings and risks associated with the process and thus enables the application of relevant preventive and corrective measures.

Keywords: beginning of training, supervision, psychotherapists, emotional experience, academic training.

\section{INTRODUCCIÓN}

El desarrollo profesional es especialmente difícil y retador para los terapeutas que están en las primeras etapas del entrenamiento práctico (De Stefano et al., 2007; Kuyken, Peters, Power, \& Lavender, 2003). Los estudiantes perciben el inicio de la formación profesional como emocionante y desafiante a la vez, ya que se pueden ver impactados y abrumados por elementos como los altos estándares de desempeño, expectativas poco realistas, la orientación al logro de la academia, el miedo de ser inadecuados para el trabajo o carentes de conocimientos y 
competencias profesionales, la preparación e incertidumbre para atender a los primeros clientes, la tarea de establecer una alianza terapéutica con el cliente, la integración de distintos esquemas teóricos, el entorno institucional y el componente evaluativo (Ronnestad \& Skovholt, 2003; Skovholt \& Ronnestad, 2003).

Al principio del entrenamiento, los terapeutas carecen naturalmente de las competencias y habilidades para lograr un desempeño adecuado en la práctica psicoterapéutica (Woodside, Oberman, Cole, \& Carruth, 2007). Debido a que hay inexperiencia y poco conocimiento teórico-práctico, se presenta una urgencia por ganar la mayor experiencia en el menor tiempo posible para comprobar que se tengan las capacidades necesarias para ejercer el rol de psicoterapeuta (Vethencourt, 2015), y para saber si se lograrán satisfacer las expectativas del programa académico (Ronnestad \& Skovholt, 2003).

Los psicoterapeutas al inicio de su formación profesional suelen estar emocionalmente sobrecargados y abrumados por sentimientos de ansiedad, inseguridad, vulnerabilidad o dudas sobre sí mismos (Proți, 2016; Watkins, 2016), algo que está relacionado específicamente a la experiencia práctica de la psicoterapia (Fitch \& Marshall, 2002; Skovholt \& Ronnestad, 2003). De hecho, la experiencia de estar cara a cara con un cliente por primera vez está llena de ansiedad, temor e incertidumbre porque los alumnos se sienten responsables sobre el bienestar de sus clientes, pero al mismo tiempo no sienten que posean las habilidades necesarias para ayudarlos y lograr un trabajo adecuado (De Stefano et al., 2007). Se ha encontrado que los sentimientos de incompetencia suelen estar presentes para la mayoría de los psicoterapeutas en su trabajo profesional (Nissen-Lie et al., 2017).

En este periodo los estudiantes también comienzan la supervisión psicoterapéutica, algo que puede ser difícil porque trae consigo niveles más altos de complejidad y ambigüedad (Skovholt \& Ronnestad, 2003). Específicamente, la supervisión psicoterapéutica puede producir en los supervisados ansiedad y preocupación por demostrar un buen rendimiento en la práctica, autoconciencia de ser observado, miedo a exponer insuficiencias personales y profesionales, y temor a una evaluación negativa que perjudique su sentido de autoestima profesional (De Stefano et al., 2007).

La manera en que los terapeutas experimentan el inicio de su entrenamiento se ha asociado con ciertos riesgos (Christopher \& Maris, 2010). Se ha hallado que el estrés resultante de esta etapa puede provocar efectos dañinos para los terapeutas y sus clientes reduciendo la eficacia y posibilidades de éxito en el trabajo psicoterapéutico, debido a que puede impactar la capacidad para prestar atención, mantener la concentración y realizar toma de decisiones efectiva (Shapiro, Shapiro, \& Schwartz, 2000). Es por esto que es relevante evaluar cómo el estrés personal de los terapeutas perjudica sus habilidades para realizar psicoterapia (Renjilian \& Stites, 2002), considerando que cuando los terapeutas están funcionando por debajo de los niveles razonables de eficacia - por ejemplo cuando están ansiosos, desinteresados o distantes - el resultado 
de la psicoterapia puede sufrir considerablemente (Campagne, 2012; Ulberg, Amlo, Hersoug, Dahl, \& Høglend, 2014).

Los terapeutas en entrenamiento deben estar conscientes de cómo puede verse afectada su eficacia profesional cuando aparecen este tipo de vivencias estresantes y negativas, deben saber cómo reconocerlas y qué hacer al respecto (Campagne, 2012), por lo que se enfatiza la importancia de que los psicoterapeutas aprendan y utilicen estrategias de autocuidado (Bradley, Whinsenhunt, Adamson, \& Kress, 2013; Brucato \& Neimeyer, 2009).

Por otro lado, se necesita mayor consciencia y conocimiento profundo por parte de los educadores y creadores de programas de formación sobre las necesidades de este grupo para ser capaces de generar posibilidades efectivas de ayudarlos (Thériault \& Gazzola, 2005). Diseñar el entrenamiento buscando disminuir los niveles de ansiedad y estrés y promoviendo el sentido de autoeficacia en psicoterapeutas ofrece la posibilitad de mejorar el rendimiento actual (Ladany, 2014; Schwoerer, May, Hollesbe, \& Mencl, 2005).

\section{MÉTODO}

\section{Participantes}

Para obtener el grupo de participantes se invitó a 15 psicoterapeutas radicados en la Ciudad de México a formar parte del estudio; no hubo criterio de exclusión en cuanto a corriente teórica, edad o sexo. El grupo de participantes estuvo conformado por 15 psicoterapeutas con rango de edad entre 22 años y 60 años, de ellos 14 fueron mujeres y uno fue hombre. Trece de los sujetos se encontraban cursando estudios de maestría y estaban en su primer año de prácticas clínicas supervisadas, los dos restantes tenían más de 10 años de experiencia en práctica clínica y ejercían el rol de supervisor para grupos de psicoterapeutas en entrenamiento.

\section{Recolección de la información}

Se llevaron a cabo dos métodos de recolección de información: entrevistas a profundidad y preguntas abiertas. Se realizaron entrevistas a profundidad a cuatro de los participantes para conocer a detalle las vivencias y opiniones de dos psicoterapeutas que cursaran en la ese momento su entrenamiento psicoterapéutico y de dos que enseñaran y supervisaran a alumnos al iniciar dicho entrenamiento. Por otro lado, se enviaron dos preguntas abiertas a 11 psicoterapeutas en entrenamiento cuyas respuestas fueron recibidas de manera escrita, por correo electrónico o en físico; esto se hizo para ampliar la perspectiva de los psicoterapeutas en entrenamiento. Tanto en las entrevistas a profundidad como en las preguntas abiertas, se indagó la perspectiva de cada participante sobre 
cómo son vivenciados o experimentados el inicio del entrenamiento formal y la supervisión.

\section{Procedimiento}

Los participantes fueron invitados por la investigadora vía contacto personal, con una explicación a detalle sobre el objetivo del estudio y lo que implicaría su participación. Con aquellos que fueron entrevistados a profundidad, se procedió a pautar la fecha de la primera entrevista y previo a la obtención de los datos se les entregó un formato de identificación y de consentimiento informado. Los participantes que respondieron las preguntas abiertas de manera escrita fueron informados del consentimiento, pero su participación fue anónima.

Las transcripciones de las entrevistas y de las respuestas fueron leídas varias veces de manera minuciosa, permitiendo un análisis exhaustivo con base en las diferencias y similitudes del contenido a través del método comparativo constante. De esta forma, se realizó la codificación abierta mediante la fragmentación de los datos en unidades discretas, es decir, en palabras, frases y oraciones, cada una de las cuales fueron consideradas como unidades de análisis. El siguiente paso consistió en la codificación axial, proceso que permitió la agrupación de las unidades de análisis según similitudes conceptuales, en categorías y subcategorías, procediendo después al etiquetado con base en códigos vivos y en la vinculación de las mismas según sus relaciones de semejanza.

\section{RESULTADOS}

El inicio del entrenamiento es vivido con la sensación de ser una invasión de temor y preocupación, del afecto sobre el pensamiento y de conocimientos prácticos y teóricos "en el primer semestre fue mucha la invasión”. Dicha invasión es inmediata, abrumadora, angustiante y hasta asfixiante y tiene que ver con la primera aproximación al rol de psicoterapeuta y con el hecho de que los conocimientos en sí mismos son invasivos.

Esta etapa también es descrita como ansiosa, "al principio con mucha ansiedad porque son los primeros pasos”, y difícil, como una actividad que cuesta trabajo, que implica mucha lucha y que conlleva una cantidad de retos y obstáculos, "da la sensación de estar caminando cuesta arriba", algo que tiene que ver con "la dificultad de andar por un sendero que es pedregoso". Entrenarse es descrito como un proceso que toma mucho tiempo y que implica estar "a la espera de que algo pase", por lo que es visto como "una espera compleja y una cuestión incierta". Es por esto que, en general, el inicio del entrenamiento se describe como "un ciclo de trabajo pesadísimo”, desde cualquier enfoque teórico, considerando que para los alumnos "es pesado todo lo que ignoras, es pesado todo lo que te falta".

Específicamente se describen ciertos retos o dificultades presentes en el entrenamiento, entre los cuales se encuentran: 


\section{Dificultad de ejercer el rol de psicoterapeuta}

Ejercer el rol de psicoterapeuta es visto como una tarea complicada, "el contacto con otro a nivel de ayuda es difícil", "no es fácil lograrlo", porque "es enfrentarte al dolor ajeno de una manera muy frontal y muy profunda" e implica trabajar con el dolor humano y psíquico, con el sufrimiento, con ansiedades básicas y con conflictos humanos fuertes; eso "cuesta trabajo".

\section{Desarrollar las habilidades con los clientes}

Los terapeutas en entrenamiento se preocupan por desarrollar la habilidad de saber cómo y cuándo intervenir, de intentar soluciones distintas, de tener creatividad en las intervenciones, de saber aplicar los modelos teóricos aprendidos, de lograr una buena relación terapéutica con los clientes, de saber innovar, de poder cocrear soluciones con los clientes y de poder estar preparados para una gran variedad de problemas. Pero este proceso implica poder dominar las dudas personales sobre si se tienen las bases necesarias, "respecto a si lo lograré o no"; poder superar la inexperiencia y poder tener paciencia con los propios procesos de aprendizaje.

\section{Sobreexigencia}

La dificultad en el rol y en el manejo de la técnica y la teoría los lleva a sobreexigirse para cumplir con los requisitos básicos, "cuando empezamos a tratar de ser psicoterapeutas nos podemos sobreexigir", creando un modelo de desempeño un tanto exigente y difícil de alcanzar. Además, sienten una exigencia importante por parte del ámbito académico donde se desenvuelven "hay una exigencia del manejo de muchas estrategias de intervención..., del manejo de la técnica".

\section{Lidiar con la pesada carga de trabajo y de estudio}

Lidiar con la pesada carga de trabajo y de estudio es visto como uno de los retos más complicados, ya que "la competencia de actividades, tareas y compromisos te lleva a un momento de duda", pues hay que dedicarle mucho tiempo a estudiar y trabajar en el aspecto teórico, dar rendimiento académico, investigar sobre los casos y estar en constante capacitación para manejar las diversas problemáticas que traigan los clientes.

\section{La supervisión}

La supervisión es vista como difícil tanto en formato individual como grupal, ya que los alumnos sienten que no cuentan con la suficiente información o experiencia para recibir y dar sugerencias y por el hecho de 
que les cuesta adaptarse a los distintos estilos y formas de supervisión y que conlleva la dedicación de un tiempo considerable.

\section{Trabajar en ti}

Los terapeutas en entrenamiento consideran que es necesario llevar un proceso terapéutico personal, pero esto es visto con cierta dificultad porque implica "una confrontación con tu self" y trabajar en uno mismo "aprendiendo a confiar en las propias capacidades, a navegar, a superar lo que te da ansiedad, miedo y lo que te frustra”.

\section{Te afecta}

El comenzar a ver clientes afecta a los terapeutas en entrenamiento porque los lleva a enfrentarse a "historias de mucho dolor y desgaste emocional"; los afecta, los conmueve y los hace sentirse mal y angustiados debido a la conexión profunda que establecen con los clientes y con la naturaleza de los casos, "el tipo de historias que escuchas hacen que pierdas piso, te queman". Esto produce una sobrecarga que los lleva a que continúen pensando en sus clientes aun cuando ya no los estén viendo, algo que es visto como bastante común para este grupo, "cargas sus historias porque saliendo voy pensando cómo intervenir la próxima sesión”, “te encariñas con las personas" y "me conmueve tanto la situación que me lo quiero llevar a mi casa" porque "el hecho de conectar con otro ser humano te llega a conmover" y porque "la ansiedad y la emoción se los traga".

\section{Sobreinvolucrarse}

La afectación emocional puede tener repercusiones más allá de un estado emocional negativo en el terapeuta en entrenamiento, ya que los terapeutas se focalizan más en sus sentimientos que en los de sus clientes, "conectan tanto con el paciente que no pueden realmente serles útiles", pues "en ese afán de establecer una alianza y ser empáticos se convierten en uno con el paciente", es decir, "es tanta la empatía que se vuelve simpatía". Por lo tanto, un reto a superar es el de evitar sobreinvolucrarse con los clientes, algo que además es visto como un gran riesgo y que implica vincularse más de lo que profesionalmente es recomendable o "convertirse en uno con el cliente", algo que puede suceder, ya que "se adentran tanto en el paciente que salen de su rol psicoterapéutico" y se convierte en un tema personal, lo que les puede nublar la mirada y hacer que pierdan objetividad, abriendo la posibilidad de "meterse en problemas" y no ser útiles a los clientes.

\section{Creerse Superman}

Los terapeutas reportan que un aspecto que puede hacer más pesado el inicio del entrenamiento tiene que ver con un "narcisismo" que 
puede generar dificultad al trabajar con los clientes, "estoy creyéndome Superman" y "si te quedas aferrado a eso te nublas". Esto genera que dejen de cuidarse a sí mismos para evitar condiciones como el Síndrome Burnout o el sobreinvolucramiento con clientes "uno de los riesgos más importantes es que los terapeutas no se cuidan" y "los propios conflictos no trabajados te pueden llevar a sobreinvolucrarte o a realizar un trabajo no ético".

\section{Atender a los primeros clientes}

"Pensé que ver pacientes iba a ser más sencillo y fluido pero la verdad fue otra". Comenzar a ver clientes es descrito como una mezcla de emociones, en la cual los terapeutas se sienten emocionados y "grandes por haber logrado lo que alguna vez admiré en otros", pero a la vez con mucha ansiedad, nerviosismo, dudas y temor.

\section{Ansiedad y nerviosismo}

Cuando se ven los primeros clientes se siente mucha ansiedad y nerviosismo, específicamente de cometer errores, de "hacer las cosas mal", "de hacerle daño a algún paciente", de "regarla" y "de no ser la adecuada". Comentan que esta ansiedad es intensa, "me dio pánico entrar al consultorio", "una noche antes no pude dormir bien" y que provoca que la experiencia no sea placentera, "en mi primer día de prácticas más que disfrutarlo estaba sufriendo". Esta ansiedad puede aumentar las probabilidades de cometer errores porque muchas veces los alumnos no saben cómo lidiar con ésta, "no podía hacer nada". Esto se relaciona con el sentimiento de no saber qué hacer, no saber qué preguntar, qué decir, cómo decirlo ni qué recomendar que muchos reportan, “¿cómo hago las preguntas?, ¿qué hago?, ¿qué le pregunto?”

\section{Dudas sobre símismo}

El no saber qué hacer puede a su vez tener que ver con dudas y cuestionamientos que los alumnos se hacen sobre sus propias capacidades, conocimientos, sobre si podrán cumplir con las expectativas de los pacientes y si podrán ayudarlos. En este grupo aparecen expectativas negativas, "pensé que no podría", ya que muchos comentan que no se sienten listos o que "no nos han dado suficiente de la técnica" y esto genera que con los clientes no se sientan adecuados o capaces, "pensé que era muy pequeña y que los clientes no confiarían en mí" y "en ocasiones sentía que lo que había aprendido había desaparecido". Ante esa sensación de incapacidad profesional e inexperiencia, sintieron "temor a que el paciente se diera cuenta de que no tenía la menor idea de lo que estaba haciendo", ya que "me sentía muy inexperta y pensé que el cliente iba a darse cuenta de mi inexperiencia". 


\section{Cuando algún cliente deja de asistir a psicoterapia}

Los terapeutas en entrenamiento pueden sentirse de valorados y pensar que la terminación abrupta o el abandono del tratamiento tiene que ver con fallas propias. Sienten que "les pega en el narcisismo" y que es algo difícil de aceptar, "no puede ser", pero que "tienes que intentar aceptarlo". Por otro lado, se atribuyen toda la responsabilidad de la terminación del tratamiento, "se me ocurren muchísimas cosas en fallas mías", tales como "a lo mejor estaba pensando en teoría" "a lo mejor interpreté muy rápido", "a lo mejor me falló interpretar alguna cosa", "a lo mejor no estaba siendo empática" y "a lo mejor no trabajé bien o vi cuestiones diferentes".

Las dificultades asociadas al entrenamiento, la ansiedad, las dudas sobre sí mismos, el sobreinvolucrarse, el no saber qué hacer y el hecho de atribuirse toda la responsabilidad son explicados por la inexperiencia normal de los terapeutas en entrenamiento, "de esa inexperiencia derivan muchas cosas porque son muy jóvenes y les falta experiencia de vida", por lo que todas estas vivencias "vienen con el empezar y con el ser novicio". Por su parte, la ansiedad, el riesgo de caer en Sindrome de Burnout, la falta de seguridad y la dificultad percibida de la formación también se deben a la inexperiencia. Específicamente el sobreinvolucrarse tiene que ver con un "no saber cómo separar las emociones" ni "poder dimensionar" debido a la falta de experiencia. Asimismo, "al principio la inexperiencia te puede hacer pensar que tiene que ver más contigo que con el paciente y hacer que lo centres más en ti" por lo que la derrota y las dudas sobre el propio desempeño se relacionan con no saber por ser nuevo en el rol.

Además de la inexperiencia, las dificultades y estados emocionales negativos asociados al inicio del entrenamiento, tiene que ver con las características propias de los terapeutas en entrenamiento, quienes "apenas se están entrenando" y "son estudiantes que empiezan a conocer el mundo de la psicoterapia pero que todavía no saben mucho de él” y están el proceso de aprender a ejercer el rol, "están aprendiendo nuevas habilidades y sensibilidades", "están aprendiendo que sus destrezas pueden hacer ciertas cosas y se está perfeccionando un poco más”. Al principio parece ser normal que no confíen en "su capacidad humana para entender al otro", que "pierdan el foco", "que no se cuestionen y se vayan con una lectura literal de las situaciones", que "no estén muy conscientes de los riesgos", que "no puedan dimensionar" y que a pesar de todo esto tengan "una genuina necesidad de ayudar" que los impulsa a seguir a pesar de todas las dificultades y vivencias emocionales negativas asociadas al inicio del entrenamiento formal.

\section{DISCUSIÓN}

Es relevante estudiar las vivencias emocionales relacionadas con ansiedad y estrés en psicoterapeutas durante el inicio de su entrenamiento, para poder evaluar el efecto que las mismas pueden tener sobre el desempeño profesional (Campagne, 2012; Renjilian \& Stites, 2002; Shapiro et al., 2000). El interés para hacer esta investigación surgió porque este 
tema ha recibido insuficiente atención, se necesita mayor conocimiento y conciencia por parte de los educadores y creadores de programas de formación sobre las necesidades de este grupo para ser capaces de ayudarlos (Thiérault \& Gazzola, 2005). El presente estudio, que forma parte de una investigación doctoral, tuvo como objetivo entender desde la perspectiva de terapeutas en entrenamiento y de supervisores/docentes cómo se vive el inicio del entrenamiento psicoterapéutico.

De los resultados obtenidos se puede observar que, tal como se constató en la literatura, el inicio del entrenamiento es vivido como una etapa difícil, por la alta y pesada carga de trabajo que implica, que involucra mucha lucha, retos y dificultades; que toma tiempo, que está llena de ansiedad e incertidumbre y que requiere de un trabajo personal. Estos elementos, según Ronnestad y Skovholt (2003) y Skovholt y Ronnestad (2003), llevan al aprendiz de terapeuta a sentirse impactado y abrumado, sensación que describen los participantes como de invasión inmediata de temor y preocupación, algo que les genera ansiedad generalizada y una sensación de sentirse asfixiados.

Dicha ansiedad parece estar relacionada con lo reportado por Woodside et al. (2007) y Fitch y Marshall (2002) sobre el temor a ser inadecuados para el rol, la sensación de que son incapaces y que no están calificados para la tarea y la percepción del alto nivel de dificultad de la práctica psicoterapéutica. Esto se observa en la evaluación que los participantes realizan sobre lo difícil que consideran que es ejercer el rol psicoterapéutico, desarrollar las habilidades necesarias para lidiar con los clientes, y poder cumplir con sus expectativas de ayuda. En la medida en que existan temores por parte de los terapeutas en entrenamiento sobre sus competencias profesionales, será más difícil para ellos sentirse adecuados para el trabajo (Ronnestad \& Skovholt, 2003; Skovholt \& Ronnestad, 2003).

Las dificultades relacionadas a comenzar a ejercer el rol de psicoterapeuta se pueden observar específicamente en los sentimientos presentes a la hora de atender a los primeros pacientes; se halló que esto implica una mezcla de emociones que incluyen emoción, ansiedad, nerviosismo, dudas y temor por cometer errores, y que en general se vive con mucha mayor dificultad de la esperada. Esto se relaciona con lo hallado por De Stefano et al. (2007) sobre el hecho de que la experiencia de estar cara a cara con un cliente por primera vez está llena de ansiedad, temor e incertidumbre y se convierte en un momento intenso y complejo para los estudiantes.

Puede ser que la experiencia de atender clientes por primera vez se vea empeorada por el hecho de que los psicoterapeutas en entrenamiento sostienen altos estándares de desempeño, expectativas poco realistas y una urgencia por demostrar el cumplimiento de esas expectativas (Ronnestad \& Skovholt, 2003; Skovholt \& Ronnestad, 2003; Vethencourt, 2015). Esto fue corroborado en los hallazgos de esta investigación en las sobre exigencias personal y académica que llevan a los estudiantes a generar modelos de desempeño rigurosos y difíciles de alcanzar. La sobre evaluación del propio alcance los lleva a no saber dimensionar y 
a pensar que todo en la relación psicoterapéutica depende de ellos y de las fallas que ellos puedan llegar a cometer, atribuyéndose así el total de la responsabilidad y por ende aumentando la posibilidad de percibir y experimentar fracasos en la práctica clínica.

Un factor que es significativo dentro del proceso de iniciar el entrenamiento y que aumenta el nivel de dificultad y frustración, es el enfrentarse a la supervisión, la cual es vista como una tarea nueva que involucra niveles altos de complejidad y que lleva consigo la evaluación y calificación de las habilidades psicoterapéuticas (De Stefano et al., 2007; Skovholt \& Ronnestad, 2003). Esto es confirmado por los participantes, quienes reportan que la supervisión es percibida como difícil, y ante la cual se sienten insuficientes por no saber cómo adaptarse a ella y cómo cumplir con los requerimientos implícitos, tales como aceptar y ofrecer sugerencias y observaciones sobre el desempeño clínico propio y de otros.

Las dificultades antes descritas se han asociado a ciertos riesgos, tales como lo establecen Christopher y Maris (2010); dichos riesgos se ven ampliados con los resultados de esta investigación e incluyen a) la afectación o sobrecarga emocional que experimentan los estudiantes al enfrentarse por primera vez a clientes y sus historias, así como a la pesada carga de trabajo inherente al entrenamiento ("la ansiedad se los traga"); y b) el sobreinvolucramiento que puede darse hacia los clientes, una situación que surge debido a una conexión más profunda de lo necesario y que los puede llevar a perder la objetividad como psicoterapeutas y hacer que dejen de ser útiles en su rol de ayuda. Sabemos por Shapiro et al. (2000) que el estrés que resulta de las situaciones emocionales antes descritas puede reducir la eficacia de los terapeutas en su trabajo, además pudiera llevarlos incluso al abuso de sustancias o problemas en su vida personal.

Considerando lo hallado en esta investigación relacionado a que altos niveles de ansiedad y nerviosismo pueden aumentar la probabilidad de cometer errores en la práctica clínica y que el agotamiento emocional o burnout se asocia con un menor sentido de agencia en los psicoterapeutas (Kim et al., 2018), se confirma la relevancia resaltada por Campagne (2012) y Renjilian y Stites (2002) de estudiar la manera en que el estrés personal de los terapeutas perjudica sus habilidades para realizar psicoterapia.

Es necesario divulgar y concientizar la vivencia emocional de psicoterapeutas durante el inicio de su entrenamiento para generar posibilidades de concientización sobre los efectos que la misma puede tener en su desempeño profesional. El que esta vivencia emocional sea normalizada, les permitiría a los terapeutas en entrenamiento y a los docentes entender sus consecuencias y aplicar estrategias de afrontamiento y autocuidado (Bradley et al., 2013; Campagne, 2012; Zeeck et al., 2012).

Con respecto a esto, se han generado críticas con respecto a la insuficiencia de la mayoría de los programas de formación para tomar en cuenta las necesidades emocionales de sus alumnos, sea en la supervisión formal (Farber \& Hazanov, 2014), en la enseñanza de estrategias de 
autocuidado y manejo de sentimientos negativos (Christopher \& Maris, 2010), o en el fomento de la sensación de competencia profesional (Thériault \& Gazzola, 2005; Thériault, Gazzola, \& Richardson, 2009).

Necesitamos desarrollar una comprensión más exhaustiva sobre cómo la etapa de desarrollo profesional puede incidir en el estado emocional de los psicoterapeutas (Råbu, Moltu, Binder, \& McLeod, 2016), ya que la capacidad de los mismos para enfrentar y manejar dificultades es relevante para la efectividad profesional (Skovholt, 2012; Zeeck et al., 2012).

Conocer más sobre los predictores del agotamiento emocional en psicoterapeutas ayudará a los supervisores y docentes a seleccionar, sensibilizar y enseñar a los alumnos de manera diferencial (Rzeszutek \& Schier, 2014). Con resultados como los de esta investigación se puede obtener una comprensión más completa sobre la vivencia emocional subjetiva de los psicoterapeutas y si se obtiene un mayor entendimiento sobre el desarrollo profesional de terapeutas, entonces se pueden establecer modelos de enseñanza y aprendizaje más eficaces, así como demandas más realistas en el entrenamiento en general.

\section{REFERENCIAS}

Bradley, N., Whinsenhunt, J., Adamson, N., \& Kress, V. (2013). Creative approaches for promoting counselor self-care. Journal of Creativity in Mental Health, 8(4), 456-469. https://doi.org/10.1080/15401383.2013 .844656

Brucato, B., \& Neimeyer, G. (2009). Epistemology as a predictor of psychotherapists' self-care and coping. Journal of Constructivist Psychology, 22(4), 269-282. https://doi.org/10.1080/10720530903113805

Campagne, D. (2012). When therapists run out of steam: Professional boredom or burnout? Revista de Psicopatología y Psicología Clínica, 17(1), 75-85.

Christopher, J., \& Maris, J. (2010). Integrating mindfulness as self-care into counselling and psychotherapy training. Counselling and Psychotherapy Research, 10(2), 114-125. https://doi.org/10.1080/1473314100375028 5

De Stefano, J., D’Iuso, N., Blake, E., Fitzpatrick, M., Drapeau, M., \& Chamodraka, M. (2007). Trainees' experiences of impasses in counseling and the impact of group supervision on their resolution: A pilot study. Counselling and Psychotherapy Research, 7(1), 42-47. https://doi.org/10. 1080/14733140601140378

Farber, B. A., \& Hazanov, V. (2014). Informal sources of supervision in clinical training. Journal of Clinical Psychology, 70(11), 1062-1072. https://doi.o $\mathrm{rg} / 10.1002 /$ jclp.22127

Fitch, T., \& Marshall, J. (2002). Using cognitive interventions with counseling practicum students during group supervision. Counselor Education and Supervision, 41, 335-342. https://doi.org/10.1002/j.1556-6978.2002.tb0 1295.x

Kim, J. J., Brookman-Frazee, L., Gellatly, R., Stadnick, N., Barnett, M. L., \& Lau, A. S. (2018). Predictors of burnout among community therapists in the sustainment phase of a system-driven implementation of multiple evidence-based practices in children's mental health. Professional 
Psychology: Research and Practice, 49(2), 132-141. http://dx.doi.org/10.1 037/pro0000182

Kuyken, W., Peters, E., Power, M., \& Lavender, T. (2003). Trainee clinical psychologists' adaptation and professional functioning: A longitudinal study. Clinical Psychology \& Psychotherapy, 10(1), 41-54. https://doi.org /10.1002/cpp.350

Ladany, N. (2014). The ingredients of supervisor failure. Journal of Clinical Psychology, 70(11), 1094-1103. https://doi.org/10.1002/jclp.22130

Nissen-Lie, H. A., Rønnestad, M. H., Høglend, P. A., Havik, O. E., Solbakken, O. A., Stiles, T. C., \& Monsen, J. T. (2017). Love yourself as a person, doubt yourself as a therapist? Clinical Psychology and Psychotherapy, 24(1), 48-60. https://doi.org/10.1002/cpp.1977

Proți, L. E. (2016). Challenges encountered by psychotherapists at the beginning of their practice. Romanian Journal of Cognitive Behavioral Therapy and Hypnosis, 3(1), 5-10.

Råbu, M., Moltu, C., Binder, P., \& McLeod, J. (2016). How does practicing psychotherapy affect the personal life of the therapist? A qualitative inquiry of senior therapists' experiences. Psychotherapy Research, 26(6), 737-749. https://doi.org/10.1080/10503307.2015.1065354

Renjilian, D., \& Stites, J. (2002). Perception of therapist burnout by college students with and without prior counseling experience. Journal of College Student Psychotherapy, 17(1), 7-18. https://doi.org/10.1300/J035v17n0 1_03

Ronnestad, M., \& Skovholt, T. (2003). The journey of the counselor and therapist: Research findings and perspectives on professional development. Journal of Career Development, 30(1), 5-44. https://doi.org /10.1023/A:1025173508081

Rzeszutek, M., \& Schier, K. (2014). Temperament traits, social support, and burnout symptoms in a sample of therapists. Psychotherapy, 51(4), 574-579. http://dx.doi.org/10.1037/a0036020

Schwoerer, C., May, D., Hollesbe, E., \& Mencl, J. (2005). General and specific self-efficacy in the context of a training intervention to enhance performance expectancy. Human Resource Development Quarterly, 16(1), 111-129. https://doi.org/10.1002/hrdq.1126

Shapiro, S., Shapiro, D., \& Schwartz, G. (2000). Stress management in medical education: A review of the literature. Academic Medicine, 75(7), 748-759.

Skovholt, T., \& Ronnestad, M. (2003). Struggles of the novice counselor and therapist.Journal of Career Development, 30(1), 45-58. https://doi.org/1 $0.1177 / 089484530303000103$

Skovholt, T. M. (2012). The counselor's resilient self.Turkish Psychological Counseling and Guidance Journal, 4(38), 137-146.

Thériault, A., \& Gazzola, N. (2005). Feelings of inadequacy, insecurity, and incompetence among experienced therapists. Counselling and Psychotherapy Research, 5(1), 11-18. https://doi.org/10.1080/14733140 512331343840

Thériault, A., Gazzola, N., \& Richardson, B. (2009). Feelings of incompetence in novice therapists: Consequences, coping, and correctives. Canadian Journal of Counselling and Psychotherapy/Revue Canadienne de Counseling et de Psychothérapie, 43(2), 105-119. 
Ulberg, R., Amlo, S., Hersoug, A. G., Dahl, H. S., \& Høglend, P. (2014). The effects of the therapist's disengaged feelings on the in-session process in psychodynamic psychotherapy. Journal of Clinical Psychology: In Session, 70(5), 440-451. https://doi.org/10.1002/jclp.22088

Vethencourt, V. (2015). Bájate de tu corcel blanco: Reflexiones sobre el fracaso en psicoterapia (Tesis de maestría). Universidad de las Américas D.F., México.

Watkins, C. E. (2016). Listening, learning, and development in psychoanalytic supervision: A self psychology perspective. Psychoanalytic Psychology, 33(3), 437-471. http://dx.doi.org/10.1037/a0038168

Woodside, M., Oberman, A., Cole, K., \& Carruth, E. K. (2007). Learning to be a counselor: A prepracticum point of view. Counselor Education and Supervision, 47, 14-28. https://doi.org/10.1002/j.1556-6978.2007.tb000 35. $\mathrm{x}$

Zeeck, A., Orlinsky, D. E., Hermann, S., Joos, A., Wirsching, M., Weidmann, W., \& Hartmann, A. (2012). Stressful involvement in psychotherapeutic work: Therapist, client and process correlates. Psychotherapy Research, 22(5), 543-555. http://dx.doi.org/10.1080/10503307.2012.683345 